\title{
Author Index Volume 27 (2014)
}

The issue number is given in front of the pagination

Abbasi, L., see Kamali, F. (4) 475-480

Abbaszadeh, S., see Wand, B.M. (4) 463-473

Abdel-aziem, A.A. and A.H. Draz, Chronic ankle instability alters eccentric eversion/inversion and dorsiflexion/plantarflexion ratio (1) 47-53

Abdel-aziem, A.A., see Mohammad, W.S. (2) 147-152

Abdelraouf, O.R., see Mohammad, W.S. (2) 147-152

Adsuar, J.C., see del Pozo-Cruz, B. (4) 399-408

Aghayev, E., see Melloh, M. (3) 281-289

Ahmadi, A., see Salahzadeh, Z. (2) 131-139

Ahmadi, M.H., see Darzi, M.T. (4) 507-512

Akarirmak, U., see Sari, H. (2) 125-129

Akbari, M., see Moradi, Z. (3) 299-306

Akgün, K., see Özçakar, L. (2) 117-124

Akkaya, N., see Özçakar, L. (2) 117-124

Akyol, Y., see Durmus, D. (4) 435-443

Alayli, G., see Durmus, D. (4) 435-443

Al-Ghadir, A.H., see Shakil, H. (2) 111-115

Alizadeh, M.H., see Seidi, F. (1) 7-16

Altay, Z., see Özçakar, L. (2) 117-124

Altindag, O., see Gur, A. (2) 239-245

Álvarez-Álvarez, S., F. García-Muro San José, A.L. Rodríguez-Fernández, J. Güeita-Rodríguez and B.J. Waller, Effects of Kinesio ${ }^{\circledR}$ Tape in low back muscle fatigue: Randomized, controlled, doubled-blinded clinical trial on healthy subjects (2) 203-212

Amorim, C.F., see Parreira, R.B. (2) 153-159

Ansari, N.N., see Moradi, Z. (3) 299-306

Arab, A.M. and M.R. Nourbakhsh, Hamstring muscle length and lumbar lordosis in subjects with different lifestyle and work setting: Comparison between individuals with and without chronic low back pain (1) 63-70

Arab, A.M., see Tahan, H. (3) 339-347

Arellano-Hernández, A., see Nava-Bringas, T.I. (1) 4146

Arellano-Hernández, A., see Nava-Bringas, T.I. (3) 371-376
Armstrong, S.M., see Sly, E. (4) 445-452

Atan, Ç., see Özçakar, L. (2) 117-124

Avendaño-Coy, J., J. Gómez-Soriano, M. Valencia, J. Estrada, F. Leal and R. Ruiz-Campa, Botulinum toxin type A and myofascial pain syndrome: A retrospective study of 301 patients (4) 485-492

Aydın, Y., see Çıtak Karakaya, I. (2) 191-196

Aydin, T., see Demir, S.E. (2) 167-171

Aygül, R., D. Kotan, H. Ulvi, M. Kuyucu, G. Özdemir, A. Ertekin and F.Ö. Odabaş, The relationship of median nerve F-wave parameters with severity and subtypes of carpal tunnel syndrome (1) 1-6

Aytekin, E., see Demir, S.E. (2) 167-171

Babaki, A.E.S., see Rostami, M. (2) 213-221

Bahrami, F., see Hedayati, R. (1) 33-40

Bardak, A., see Özçakar, L. (2) 117-124

Barz, T., see Melloh, M. (3) 281-289

Basar, S., I. Duzgun, N.A. Guzel, I. Cicioğlu and B. Çelik, Differences in strength, flexibility, and stability in freestyle and Greco-Roman wrestlers (3) 321-330

Battié, M.C., see Tomkins-Lane, C.C. (2) 223-229

Baysal, Ö., see Özçakar, L. (2) 117-124

Bedekar, N., M. Suryawanshi, S. Rairikar, P. Sancheti and A. Shyam, Inter and Intra-rater reliability of mobile device goniometer in measuring lumbar flexion range of motion (2) 161-166

Been, E., see Bezalel, T. (4) 383-390

Behtash, H., see Salahzadeh, Z. (2) 131-139

Beneka, A., see Malliou, P. (3) 361-370

Berral, F.J., see Oyarzo, C.A. (2) 141-146

Bezalel, T., E. Carmeli, E. Been and L. Kalichman, Scheuermann's disease: Current diagnosis and treatment approach (4) 383-390

Bilgici, A., see Özçakar, L. (2) 117-124

Bilgici, A., see Durmus, D. (4) 435-443

Bozkurt, M., see Cayir, A. (3) 307-313

Briffa, K.N., see Schäfer, A.G.M. (4) 409-418 
Buğdaycı, D., see Özçakar, L. (2) 117-124

Çapkın, E., see Özçakar, L. (2) 117-124

Çarlı, A.B., see Özçakar, L. (2) 117-124

Carmeli, E., see Bezalel, T. (4) 383-390

Carpintero, P., see Oyarzo, C.A. (2) 141-146

Cayir, A., G. Yavuzer, R.T. Sayli, E. Gurcay, V. Culha and M. Bozkurt, Evaluation of joint findings with gait analysis in children with hemophilia (3) 307313

Çelik, B., see Basar, S. (3) 321-330

Chandra, A.M., see Maulik, S. (4) 453-461

Cicholas, B., see Linden, M. (4) 563-568

Cicioğlu, I., see Basar, S. (3) 321-330

Ciftkaya, P.O., see Civelek, G.M. (4) 537-544

Çıtak Karakaya, İ., Ö.F. Güney, Y. Aydın and M.G. Karakaya, Effects of thermal agents on electrical sensory threshold and current tolerance when applied prior to neuromuscular electrical stimulation (2) 191-196

Çıtak Karakaya, İ., M.G. Karakaya, E. Erğun, S. Elmalı and T. Firat, Effects of different frequencies of conventional transcutaneous electrical nerve stimulation on pressure pain threshold and tolerance (2) 197-201

Civelek, G.M., P.O. Ciftkaya and M. Karatas, Evaluation of restless legs syndrome in fibromyalgia syndrome: An analysis of quality of sleep and life (4) 537-544

Coleman, C., see Sly, E. (4) 445-452

Coronado-Zarco, R., see Nava-Bringas, T.I. (1) 41-46

Coronado-Zarco, R., see Nava-Bringas, T.I. (3) 371376

Cortés Godoy, V., T. Gallego Izquierdo, I. Lázaro Navas and D. Pecos Martín, Effectiveness of massage therapy as co-adjuvant treatment to exercise in osteoarthritis of the knee: A randomized control trial (4) 521-529

Cruz-Medina, E., see Nava-Bringas, T.I. (1) 41-46

Cruz-Medina, E., see Nava-Bringas, T.I. (3) 371-376

Culha, V., see Cayir, A. (3) 307-313

da Silva, R.A., see Parreira, R.B. (2) 153-159

Dadian, M., see Darzi, M.T. (4) 507-512

Darzi, M.T., S. Pourhadi, S. Hosseinzadeh, M.H. Ahmadi and M. Dadian, Comparison of quality of life in low back pain patients and healthy subjects by using WHOQOL-BREF (4) 507-512

Davarian, S., N. Maroufi, E. Ebrahimi, M. Parnianpour and F. Farahmand, Normal postural responses preceding shoulder flexion: Co-activation or asymmetric activation of transverse abdominis? $545-551$
Davidson, M., see Iles, R.A. (1) 93-101

De Benedittis, S., see Di Sante, L. (1) 103-106

de Oliveira, M.R., see Parreira, R.B. (2) 153-159

de Souza Costa, A.C., see Packer, A.C. (4) 493-498

De, A., see Maulik, S. (4) 453-461

Dede, I., see Kesikburun, S. (3) 275-279

del Pozo-Cruz, B., M.H. Mocholi, J. del Pozo-Cruz, J.A. Parraca, J.C. Adsuar and N. Gusi, Reliability and validity of lumbar and abdominal trunk muscle endurance tests in office workers with nonspecific subacute low back pain (4) 399-408

del Pozo-Cruz, J., see del Pozo-Cruz, B. (4) 399-408

Demir, S.E., E. Aytekin, I. Karacan, T. Aydin and G.D. Kavadar, The prevalence of fibromyalgia among patients with cervical radiculopathy due to cervical disc herniation: A pilot study (2) 167-171

Deng, S.-L., see Pan, X.-M. (4) 499-505

Di Sante, L., M. Paoloni, S. De Benedittis, L. Tognolo and V. Santilli, Groin pain and iliopsoas bursitis: Always a cause-effect relationship? (1) 103-106

Dibai-Filho, A.V., see Packer, A.C. (4) 493-498

Dickens, J.C.W., see Roberts, A.J. (2) 173-179

Dıraçoğlu, D., see Özçakar, L. (2) 117-124

Doğu, B., see Özçakar, L. (2) 117-124

Doğu, B., see Yilmaz, F. (4) 391-397

dos Santos Berni, K.C., see Packer, A.C. (4) 493-498

Dost, G., D. Dulgeroglu, A. Yildirim and N. Ozgirgin, The effects of upper extremity progressive resistance and endurance exercises in patients with spinal cord injury (4) 419-426

Draz, A.H., see Abdel-aziem, A.A. (1) 47-53

Dulgeroglu, D., see Dost, G. (4) 419-426

Duman, I., see Yavuz, F. (3) 315-320

Durmuş, B., see Özçakar, L. (2) 117-124

Durmus, D., Y. Ulus, G. Alayli, Y. Akyol, A. Bilgici, K. Yazicioglu and O. Kuru, Does microwave diathermy have an effect on clinical parameters in chronic low back pain? A randomized-controlled trial (4) 435-443

Durmus, D., M. Unal and O. Kuru, How effective is a modified exercise program on its own or with back school in chronic low back pain? A randomizedcontrolled clinical trial (4) 553-561

Duzgun, I., see Basar, S. (3) 321-330

Ebrahimi, E., see Davarian, S. (4) 545-551

Ebrahimi, I., see Seidi, F. (1) 7-16

Ebrahimi, S., see Kamali, F. (4) 475-480

Efstathiou, M., see Savva, C. (4) 377-382

Elfering, A., see Melloh, M. (3) 281-289

Elmalı, S., see Çıtak Karakaya, İ. (2) 197-201 
Emrani, A., see Moradi, Z. (3) 299-306

Erden, G., see Özçakar, L. (2) 117-124

Erğun, E., see Çıtak Karakaya, İ. (2) 197-201

Erhan, B., see Özçakar, L. (2) 117-124

Ertekin, Ö., see Unver, B. (1) 77-84

Ertekin, A., see Aygül, R. (1) 1-6

Estrada, J., see Avendaño-Coy, J. (4) 485-492

Ezeakunne, A.C., see Ummunah, J.O. (3) 291-298

Fan, L., see Pan, X.-M. (4) 499-505

Farahmand, F., see Davarian, S. (4) 545-551

Ferry, R.G., see Roberts, A.J. (2) 173-179

Fırat, T., see Çıtak Karakaya, I. (2) 197-201

Formby, P.M., see Wand, B.M. (4) 463-473

Gallego Izquierdo, T., see Cortés Godoy, V. (4) 521529

García-Muro San José, F., see Álvarez-Álvarez, S. (2) 203-212

Garvey, C., see Sly, E. (4) 445-452

George, P.J., see Wand, B.M. (4) 463-473

Giakas, G., see Savva, C. (4) 377-382

Gioftsidou, A., see Malliou, P. (3) 361-370

Godolias, G., see Malliou, P. (3) 361-370

Gohari, M., see Salahzadeh, Z. (2) 131-139

Göktepe, S., see Kesikburun, S. (3) 275-279

Gomez-Hassan, D., see Sonagere, M.B. (1) 107-110

Gómez-Soriano, J., see Avendaño-Coy, J. (4) 485-492

Gopal, H. and C. McCrory, Coccygodynia treated by pulsed radio frequency treatment to the Ganglion of Impar: A case series (3) 349-354

Grabara, M., A comparison of the posture between young female handball players and non-training peers (1) 85-92

Grabara, M., Anteroposterior curvatures of the spine in adolescent athletes (4) 513-519

Güeita-Rodríguez, J., see Álvarez-Álvarez, S. (2) $203-$ 212

Gulsen, F., see Sari, H. (2) 125-129

Gun, K., see Sari, H. (2) 125-129

Gunay, S., see Yildirim, Y. (1) 25-32

Gündüz, B., see Özçakar, L. (2) 117-124

Günendi, Z., see Özçakar, L. (2) 117-124

Güney, Ö.F., see Çıtak Karakaya, İ. (2) 191-196

Gur, A., O. Altindag, H. Karagullu, I. Koca and E. Madenci, Multifocal skeletal tuberculosis presenting as paraplegia: A case report (2) 239-245

Gurcay, E., see Cayir, A. (3) 307-313

Gusi, N., see del Pozo-Cruz, B. (4) 399-408

Guzel, N.A., see Basar, S. (3) 321-330
Haig, A.J., see Sonagere, M.B. (1) 107-110

Hall, T.M., see Schäfer, A.G.M. (4) 409-418

Haun, D.W., see Nuzzo, J.L. (4) 427-433

Hedayati, R., S. Kahrizi, M. Parnianpour, F. Bahrami, A. Kazemnejad and B. Mobini, The study of the variability of anticipatory postural adjustments in patients with recurrent non-specific low back pain (1) 33-40

Hernández-López, M., see Nava-Bringas, T.I. (1) 4146

Hernández-López, M., see Nava-Bringas, T.I. (3) 371376

Hosseinzadeh, S., see Darzi, M.T. (4) 507-512

$\mathrm{Hu}, \mathrm{R}$., see Tomkins-Lane, C.C. (2) 223-229

Huang, X., see Pan, X.-M. (4) 499-505

Ibikunle, P.O., see Ummunah, J.O. (3) 291-298

Iles, R.A., N.F. Taylor, M. Davidson and P. O'Halloran, An effective coaching intervention for people with low recovery expectations and low back pain: A content analysis (1) 93-101

Iqbal, R., see Maulik, S. (4) 453-461

Iqbal, Z.A., see Shakil, H. (2) 111-115

Ishida, H. and S. Watanabe, Maximum expiration activates the abdominal muscles during side bridge exercise (4) 481-484

Ishida, H., see Watanabe, S. (1) 55-61

Jain, T.K. and N.K. Sharma, The effectiveness of physiotherapeutic interventions in treatment of frozen shoulder/adhesive capsulitis: A systematic review (3) 247-273

James, M., see Wand, B.M. (4) 463-473

Jeong, M.-K., see Kim, D.-H. (2) 231-237

Jiang, K., see Pan, X.-M. (4) 499-505

Jones, M.D., see Williams, J.M. (1) 71-75

Jones, T.G., see Roberts, A.J. (2) 173-179

Kahrizi, S., see Hedayati, R. (1) 33-40

Kalichman, L., see Bezalel, T. (4) 383-390

Kamali, F., F. Panahi, S. Ebrahimi and L. Abbasi, Comparison between massage and routine physical therapy in women with sub acute and chronic nonspecific low back pain (4) 475-480

Kaneoka, K., see Watanabe, M. (4) 531-536

Karacan, I., see Demir, S.E. (2) 167-171

Karadibak, D., see Yildirim, Y. (1) 25-32

Karagullu, H., see Gur, A. (2) 239-245

Karakaya, M.G., see Çıtak Karakaya, I. (2) 191-196

Karakaya, M.G., see Çıtak Karakaya, I. (2) 197-201

Karatas, M., see Civelek, G.M. (4) 537-544 
Karatosun, V., see Unver, B. (1) 77-84

Kavadar, G.D., see Demir, S.E. (2) 167-171

Kazemnejad, A., see Hedayati, R. (1) 33-40

Kesikburun, S., E. Yaşar, İ. Dede, S. Göktepe and A.K. Tan, Ultrasound-guided steroid injection in the treatment of stump neuroma: Pilot study (3) 275-279

Kesikburun, S., see Özçakar, L. (2) 117-124

Khademi, K., see Tahan, H. (3) 339-347

Kim, C.-Y., see Nam, S.-J. (3) 355-360

Kim, D., see Noh, D.K. (3) 331-338

Kim, D.-H., J.-K. Park and M.-K. Jeong, Influences of posterior-located center of gravity on lumbar extension strength, balance, and lumbar lordosis in chronic low back pain (2) 231-237

Kim, H., see Noh, D.K. (3) 331-338

Kim, M.-J., see Nam, S.-J. (3) 355-360

Kim, Y., H.-R. Yang, J.-W. Lee and B.-C. Yoon, Effects of the high-power pain threshold ultrasound technique in the elderly with latent myofascial trigger points: A double-blind randomized study (1) $17-23$

Ko, S.-M., see Noh, D.K. (3) 331-338

Kobara, K., see Watanabe, S. (1) 55-61

Koca, I., see Gur, A. (2) 239-245

Kocabaş, H., see Özçakar, L. (2) 117-124

Koh, J.-H., see Noh, D.K. (3) 331-338

Kordi, R., see Rostami, M. (2) 213-221

Kotan, D., see Aygül, R. (1) 1-6

Kuran, B., see Yilmaz, F. (4) 391-397

Kuru, Ö., see Özçakar, L. (2) 117-124

Kuru, O., see Durmus, D. (4) 435-443

Kuru, O., see Durmus, D. (4) 553-561

Kuyucu, M., see Aygül, R. (1) 1-6

Lázaro Navas, I., see Cortés Godoy, V. (4) 521-529

Leal, F., see Avendaño-Coy, J. (4) 485-492

Lee, J.-W., see Kim, Y. (1) 17-23

León-Hernández, S.R., see Nava-Bringas, T.I. (1) 4146

León-Hernández, S.R., see Nava-Bringas, T.I. (3) 371376

Li, W., see Pan, X.-M. (4) 499-505

Linden, M., S. Scherbe and B. Cicholas, Randomized controlled trial on the effectiveness of cognitive behavior group therapy in chronic back pain patients (4) 563-568

Lucas, D.R., see Sonagere, M.B. (1) 107-110

Lüdtke, K., see Schäfer, A.G.M. (4) 409-418

Ma, Z., see Pan, X.-M. (4) 499-505
Macedo, L., see Tomkins-Lane, C.C. (2) 223-229

Macías-Hernández, S.I., see Nava-Bringas, T.I. (1) 4146

Macías-Hernández, S.I., see Nava-Bringas, T.I. (3) 371-376

Madenci, E., see Gur, A. (2) 239-245

Malliou, P., S. Rokka, A. Beneka, A. Gioftsidou, S. Mavromoustakos and G. Godolias, Analysis of the chronic lower limb injuries occurrence in step aerobic instructors in relation to their working step class profile: A three year longitudinal prospective study (3) 361-370

Mallwitz, J., see Schäfer, A.G.M. (4) 409-418

Mansournia, M.A., see Rostami, M. (2) 213-221

Maroufi, N., see Davarian, S. (4) 545-551

Maroufi, N., see Salahzadeh, Z. (2) 131-139

Matsui, Y., see Watanabe, M. (4) 531-536

Maulik, S., R. Iqbal, A. De and A.M. Chandra, Evaluation of the working posture and prevalence of musculoskeletal symptoms among medical laboratory technicians (4) 453-461

Mavromoustakos, S., see Malliou, P. (3) 361-370

Mayer, J.M., see Nuzzo, J.L. (4) 427-433

McCormick, Z. and C. Plastaras, Transforaminal epidural steroid injection in the treatment of lumbosacral radicular pain caused by epidural lipomatosis: A case series and review (2) 181-190

McCrory, C., see Gopal, H. (3) 349-354

Melloh, M., A. Elfering, T.R. Stanton, T. Barz, E. Aghayev, C. Röder and J.-C. Theis, Low back pain risk factors associated with persistence, recurrence and delayed presentation (3) 281-289

Minoonejad, H., see Seidi, F. (1) 7-16

Miyakawa, S., see Watanabe, M. (4) 531-536

Mobini, B., see Hedayati, R. (1) 33-40

Mocholi, M.H., see del Pozo-Cruz, B. (4) 399-408

Mohammad, W.S., O.R. Abdelraouf and A.A. Abdelaziem, Concentric and eccentric strength of trunk muscles in osteitis pubis soccer players (2) 147152

Mohammadi, P., see Moradi, Z. (3) 299-306

Moradi, Z., M. Akbari, N.N. Ansari, A. Emrani and P. Mohammadi, Strength of hip muscle groups in sedentary women with patellofemoral pain syndrome (3) 299-306

Nam, S.-J., M.-J. Kim, S.-J. Yim, D.-W. Oh, H.-J. Park and C.-Y. Kim, Influence of walking speed on electromyographic activity of the rectus abdominis and erector spinae during high-heeled walking (3) 355-360 
Nava-Bringas, T.I., M. Hernández-López, I. RamírezMora, R. Coronado-Zarco, S.I. Macías-Hernández, E. Cruz-Medina, A. Arellano-Hernández and S.R. León-Hernández, Effects of a stabilization exercise program in functionality and pain in patients with degenerative spondylolisthesis (1) 41-46

Nava-Bringas, T.I., I. Ramírez-Mora, R. CoronadoZarco, S.I. Macías-Hernández, E. Cruz-Medina, A. Arellano-Hernández, M. Hernández-López and S.R. León-Hernández, Association of strength, muscle balance, and atrophy with pain and function in patients with degenerative spondylolisthesis (3) 371-376

Noh, D.K., J.-H. You, J.-H. Koh, H. Kim, D. Kim, S.M. Ko and J.-Y. Shin, Effects of novel corrective spinal technique on adolescent idiopathic scoliosis as assessed by radiographic imaging (3) 331-338

Noorian, N., see Rostami, M. (2) 213-221

Nourbakhsh, M.R., see Arab, A.M. (1) 63-70

Nuzzo, J.L., D.W. Haun and J.M. Mayer, Ultrasound measurements of lumbar multifidus and abdominal muscle size in firefighters (4) 427-433

O'Connell, N.E., see Wand, B.M. (4) 463-473

Odabaş, F.Ö., see Aygül, R. (1) 1-6

O’Donoghue, C., see Sly, E. (4) 445-452

Oh, D.-W., see Nam, S.-J. (3) 355-360

O'Halloran, P., see Iles, R.A. (1) 93-101

Öken, Ö., see Özçakar, L. (2) 117-124

Omaç, Ö.K., see Özçakar, L. (2) 117-124

Öneş, K., see Özçakar, L. (2) 117-124

Ornek, N.I., see Sari, H. (2) 125-129

Osaka, H., see Watanabe, S. (1) 55-61

Oyarzo, C.A., C.R. Villagrán, R.E. Silvestre, P. Carpintero and F.J. Berral, Postural control and low back pain in elite athletes. Comparison of static balance in elite athletes with and without low back pain (2) 141-146

Özçakar, L., H. Tunç, Ö. Öken, Z. Ünlü, B. Durmuş, Ö. Baysal, Z. Altay, F. Tok, N. Akkaya, B. Doğu, E. Çapkın, A. Bardak, A.B. Çarlı, D. Buğdaycı, H. Toktaş, D. Dıraçoğlu, B. Gündüz, B. Erhan, H. Kocabaş, G. Erden, Z. Günendi, S. Kesikburun, Ö.K. Omaç, M.A. Taşkaynatan, K. Şenel, M. Uğur, E.Y. Yalçınkaya, K. Öneş, Ç. Atan, K. Akgün, A. Bilgici, Ö. Kuru and S. Özgöçmen, Femoral cartilage thickness measurements in healthy individuals: Learning, practicing and publishing with TURK-MUSCULUS (2) $117-124$

Özdemir, G., see Aygül, R. (1) 1-6
Ozgirgin, N., see Dost, G. (4) 419-426

Özgöçmen, S., see Özçakar, L. (2) 117-124

Packer, A.C., A.V. Dibai-Filho, A.C. de Souza Costa, K.C. dos Santos Berni and D. RodriguesBigaton, Relationship between neck disability and mandibular range of motion (4) 493-498

Pan, X.-M., W. Li, X. Huang, S.-L. Deng, B. Qu, L. Fan, Z. Ma and K. Jiang, Single level anterior interbody fusion and fixation in the treatment of thoracolumbar fractures (4) 499-505

Panahi, F., see Kamali, F. (4) 475-480

Paoloni, M., see Di Sante, L. (1) 103-106

Park, H.-J., see Nam, S.-J. (3) 355-360

Park, J.-K., see Kim, D.-H. (2) 231-237

Parnianpour, M., see Davarian, S. (4) 545-551

Parnianpour, M., see Hedayati, R. (1) 33-40

Parnianpour, M., see Salahzadeh, Z. (2) 131-139

Parraca, J.A., see del Pozo-Cruz, B. (4) 399-408

Parreira, R.B., M.R. de Oliveira, C.F. Amorim, D.C. Teixeira and R.A. da Silva, Older adults present better back endurance than young adults during a dynamic trunk extension exercise (2) 153-159

Patel, R., see Sonagere, M.B. (1) 107-110

Pecos Martín, D., see Cortés Godoy, V. (4) 521-529

Plastaras, C., see McCormick, Z. (2) 181-190

Pourhadi, S., see Darzi, M.T. (4) 507-512

Qu, B., see Pan, X.-M. (4) 499-505

Quinn, D., see Sly, E. (4) 445-452

Rairikar, S., see Bedekar, N. (2) 161-166

Rajabi, R., see Seidi, F. (1) 7-16

Ramírez-Mora, I., see Nava-Bringas, T.I. (1) 41-46

Ramírez-Mora, I., see Nava-Bringas, T.I. (3) 371-376

Rasouli, O., see Tahan, H. (3) 339-347

Razmjoo, A., see Salahzadeh, Z. (2) 131-139

Reece, A.J., see Roberts, A.J. (2) 173-179

Roberts, A.J., R. Seah, J.C.W. Dickens, R.G. Ferry, A.J. Reece and T.G. Jones, A comparison of pain levels after the Biering-Sorensen and the modified 20-metre shuttle test in patients with chronic low back pain (2) 173-179

Röder, C., see Melloh, M. (3) 281-289

Rodríguez-Fernández, A.L., see Álvarez-Álvarez, S. (2) 203-212

Rodrigues-Bigaton, D., see Packer, A.C. (4) 493-498

Rokka, S., see Malliou, P. (3) 361-370

Rolke, R., see Schäfer, A.G.M. (4) 409-418

Rostami, M., N. Noorian, M.A. Mansournia, E. Sharafi, A.E.S. Babaki and R. Kordi, Validation of the Persian version of the fear avoidance belief questionnaire in patients with low back pain (2) 213-221 
Ruiz-Campa, R., see Avendaño-Coy, J. (4) 485-492

Sahin, F., see Yilmaz, F. (4) 391-397

Salahzadeh, Z., N. Maroufi, A. Ahmadi, H. Behtash, A. Razmjoo, M. Gohari and M. Parnianpour, Assessment of forward head posture in females: Observational and photogrammetry methods (2) 131139

Samani, E.N., see Tahan, H. (3) 339-347

Sancheti, P., see Bedekar, N. (2) 161-166

Santilli, V., see Di Sante, L. (1) 103-106

Sari, H., M. Uludag, U. Akarirmak, N.I. Ornek, K. Gun and F. Gulsen, Aggressive vertebral hemangioma as a rare cause of myelopathy (2) 125-129

Savva, C., G. Giakas and M. Efstathiou, The role of the descending inhibitory pain mechanism in musculoskeletal pain following high-velocity, low amplitude thrust manipulation. A review of the literature (4) 377-382

Sayli, R.T., see Cayir, A. (3) 307-313

Schäfer, A.G.M., T.M. Hall, R. Rolke, R.-D. Treede, K. Lüdtke, J. Mallwitz and K.N. Briffa, Low back related leg pain: An investigation of construct validity of a new classification system (4) 409-418

Scherbe, S., see Linden, M. (4) 563-568

Seah, R., see Roberts, A.J. (2) 173-179

Seidi, F., R. Rajabi, I. Ebrahimi, M.H. Alizadeh and H. Minoonejad, The efficiency of corrective exercise interventions on thoracic hyper-kyphosis angle (1) $7-16$

Şenel, K., see Özçakar, L. (2) 117-124

Shakil, H., Z.A. Iqbal and A.H. Al-Ghadir, Scoliosis: Review of types of curves, etiological theories and conservative treatment (2) 111-115

Sharafi, E., see Rostami, M. (2) 213-221

Sharma, N.K., see Jain, T.K. (3) 247-273

Shin, J.-Y., see Noh, D.K. (3) 331-338

Shyam, A., see Bedekar, N. (2) 161-166

Silvestre, R.E., see Oyarzo, C.A. (2) 141-146

Sirzai, H., see Yilmaz, F. (4) 391-397

Sly, E., C. Coleman, D. Quinn, S.M. Armstrong, C. Garvey, C. O'Donoghue and F. Wilson, The effect of two exercise protocols on lumbar spine sagittal range of motion (4) 445-452

Smith, A.J., see Wand, B.M. (4) 463-473

Sonagere, M.B., A.J. Haig, R. Patel, D.R. Lucas and D. Gomez-Hassan, A case study of unusual benign osseous lesion of the L5 lamina (1) 107-110

Stanton, T.R., see Melloh, M. (3) 281-289

Suryawanshi, M., see Bedekar, N. (2) 161-166
Tahan, H., O. Rasouli, A.M. Arab, K. Khademi and E.N. Samani, Reliability of the ultrasound measurements of abdominal muscles activity when activated with and without pelvic floor muscles contraction (3) 339-347

Tan, A.K., see Kesikburun, S. (3) 275-279

Tan, A.K., see Yavuz, F. (3) 315-320

Taşkaynatan, M.A., see Özçakar, L. (2) 117-124

Taskaynatan, M.A., see Yavuz, F. (3) 315-320

Taylor, N.F., see Iles, R.A. (1) 93-101

Teixeira, D.C., see Parreira, R.B. (2) 153-159

Theis, J.-C., see Melloh, M. (3) 281-289

Theobald, P.S., see Williams, J.M. (1) 71-75

Tognolo, L., see Di Sante, L. (1) 103-106

Tok, F., see Özçakar, L. (2) 117-124

Toktaş, H., see Özçakar, L. (2) 117-124

Tomkins-Lane, C.C., M.C. Battié, R. Hu and L. Macedo, Pathoanatomical characteristics of clinical lumbar spinal stenosis (2) 223-229

Treede, R.-D., see Schäfer, A.G.M. (4) 409-418

Tunç, H., see Özçakar, L. (2) 117-124

Uğur, M., see Özçakar, L. (2) 117-124

Uludag, M., see Sari, H. (2) 125-129

Ulus, Y., see Durmus, D. (4) 435-443

Ulvi, H., see Aygül, R. (1) 1-6

Ummunah, J.O., P.O. Ibikunle and A.C. Ezeakunne, Relationship between isometric endurance of back extensor muscles and selected anthropometric indices among some Nigerian undergraduates (3) 291-298

Unal, M., see Durmus, D. (4) 553-561

Ünlü, Z., see Özçakar, L. (2) 117-124

Unver, B., Ö. Ertekin and V. Karatosun, Pain, fear of falling and stair climbing ability in patients with knee osteoarthritis before and after knee replacement: 6 month follow-up study (1) 77-84

Valencia, M., see Avendaño-Coy, J. (4) 485-492

Villagrán, C.R., see Oyarzo, C.A. (2) 141-146

Wada, Y., see Watanabe, M. (4) 531-536

Waller, B.J., see Álvarez-Álvarez, S. (2) 203-212

Wand, B.M., M. James, S. Abbaszadeh, P.J. George, P.M. Formby, A.J. Smith and N.E. O'Connell, Assessing self-perception in patients with chronic low back pain: Development of a back-specific body-perception questionnaire (4) 463-473

Watanabe, M., K. Kaneoka, Y. Wada, Y. Matsui and S. Miyakawa, Trunk muscle activity with different sitting postures and pelvic inclination (4) 531-536 
Watanabe, S., K. Kobara, Y. Yoshimura, H. Osa$\mathrm{ka}$ and $\mathrm{H}$. Ishida, Influence of trunk muscle cocontraction on spinal curvature during sitting (1) $55-61$

Watanabe, S., see Ishida, H. (4) 481-484

Williams, J.M., P.S. Theobald and M.D. Jones, Does the presence of a vertical barrier influence sagittal spinal curvature or range of motion in young females? (1) 71-75

Wilson, F., see Sly, E. (4) 445-452

Yalçınkaya, E.Y., see Özçakar, L. (2) 117-124

Yang, H.-R., see Kim, Y. (1) 17-23

Yaşar, E., see Kesikburun, S. (3) 275-279

Yavuz, F., I. Duman, M.A. Taskaynatan and A.K. Tan, Low-level laser therapy versus ultrasound therapy in the treatment of subacromial impingement syndrome: A randomized clinical trial (3) 315-320
Yavuzer, G., see Cayir, A. (3) 307-313

Yazicioglu, K., see Durmus, D. (4) 435-443

Yildirim, A., see Dost, G. (4) 419-426

Yildirim, Y., S. Gunay and D. Karadibak, Identifying factors associated with low back pain among employees working at a package producing industry (1) 25-32

Yilmaz, F., B. Doğu, F. Sahin, H. Sirzai and B. Kuran, Investigation of responsiveness indices of generic and specific measures of health related quality of life in patients with osteoporosis (4) 391-397

Yim, S.-J., see Nam, S.-J. (3) 355-360

Yoon, B.-C., see Kim, Y. (1) 17-23

Yoshimura, Y., see Watanabe, S. (1) 55-61

You, J.-H., see Noh, D.K. (3) 331-338 